\title{
Effect of Low-Dose Amiodarone on Atrial Fibrillation or Flutter in Japanese Patients With Heart Failure
}

\author{
Tsuyoshi Shiga, MD; Michi Wakaumi, MD; Taku Imai, MD; \\ Tsuyoshi Suzuki, MD; Fumitaka Hosaka, MD; Yuichiro Yamada, MD; \\ Naoki Matsuda, MD; Morio Shoda, MD; Ryo Sugiura, MD; \\ Nobuhisa Hagiwara, MD; Hiroshi Kasanuki, MD
}

\begin{abstract}
The efficacy and safety of amiodarone in the management of atrial fibrillation (AF) or flutter in 108 Japanese patients with heart failure was retrospectively examined. Thirty-four $(41 \%)$ of the 82 patients who were in sinus rhythm after 1 month of amiodarone administration had their first recurrence, $70 \%$ of cases occurring within 1 year of initiation. The cumulative rates of maintenance of sinus rhythm were $0.68,0.55$, and 0.47 at 1,3 , and 5 years, respectively. Amiodarone was more effective in maintaining sinus rhythm in patients with paroxysmal AF or flutter than in those with the persistent form $(\mathrm{p}<0.05)$. The cumulative rates for cases that remained in permanent $\mathrm{AF}$ were $0.04,0.11$, and 0.14 at 1,3 , and 5 years, respectively. Apart from suppressing AF, the mean heart rate during Holter monitoring was significantly decreased with amiodarone therapy in cases of permanent AF. Adverse effects requiring the discontinuation of amiodarone therapy occurred in $16 \%$ of patients. Low-dose amiodarone therapy may prevent AF or flutter in Japanese patients with heart failure. (Circ J 2002; 66: 600-604)
\end{abstract}

Key Words: Amiodarone; Antiarrhythmic drugs; Atrial fibrillation; Atrial flutter; Heart failure

$\Delta$ rial fibrillation $(\mathrm{AF})$ frequently occurs in patients with congestive heart failure: in one study $22 \%$ of 750 heart failure patients with a left ventricular ejection fraction (LVEF) of less than $40 \%$ had a history of AF! During the 40 years of follow-up in the Framingham study, $21.8 \%$ of male and $28.9 \%$ of female subjects with $\mathrm{AF}$ developed heart failure, compared with only $3.2 \%$ and $3.7 \%$, respectively, of those without $\mathrm{AF}^{2}$. However, there are no reports concerning the incidence of $\mathrm{AF}$ or its prognosis in Japanese patients with heart failure.

Atrial fibrillation can lead to clinical deterioration and hospitalization for heart failure $3^{3-5}$ and it also increases the risk of cardiovascular morbidity among patients with a severe heart failure6 Therefore, the prevention or conversion of AF or flutter is important in the management of heart failure. Many class I antiarrhythmic agents convert $\mathrm{AF}$ and maintain sinus rhythm (SR) through a slowing of conduction velocity, but may also worsen heart failure through their significant negative inotropic effects ${ }^{7-9}$ and thus increase the risk of death in patients with heart failure? Randomized control trials for prevention of cardiac death in patients with impaired cardiac function have suggested that amiodarone therapy is suitable for management of AF in patients with heart failure, ${ }^{10-14}$ and it was reported in the recent DIAMOND trial that dofetilide was effective in converting $\mathrm{AF}$, preventing its recurrence, and reducing the risk of hospitalization for worsening heart failure in patients with congestive heat failure and left ventricular dysfunc-

(Received September 21, 2001; revised manuscript received February 26, 2002; accepted March 1, 2002)

Department of Cardiology, The Heart Institute of Japan, Tokyo Women's Medical University, Tokyo, Japan

Mailing address: Tsuyoshi Shiga, MD, Department of Cardiology,

The Heart Institute of Japan, Tokyo Women's Medical University, 8-1 Kawada-cho, Shinjuku, Tokyo 162-8666, Japan. E-mail: mshiga@hij. twmu.ac.jp tion, without affecting mortality 15 Class III antiarrhythmic drugs, such as amiodarone and dofetilide, would thus appear to have significant advantages over class I drugs in patients with $\mathrm{AF}$ and heart failure.

Amiodarone is commonly used for ventricular tachyarrhythmia in patients with underlying heart disease, but it is not always used for prevention of atrial tachyarrhythmia in patients with heart failure in Japan. There are few reports concerning the efficacy and safety of antiarrhythmic drug therapy against atrial tachyarrhythmia in Japanese patients with heart failure, so we retrospectively examined the role of amiodarone in the management of $\mathrm{AF}$ or flutter.

\section{Methods}

\section{Subjects}

The study group comprised 108 patients who received amiodarone for refractory arrhythmia at The Heart Institute of Japan, Tokyo Women's Medical University, between February 1988 and January 2001. All patients had AF or flutter and symptomatic heart failure (New York Heart Association (NYHA) functional class II-IV). All patients had underlying heart disease with an impaired left ventricular ejection fraction, estimated to be less than $50 \%$ on left ventriculography or radionuclide ventriculography. Informed consent for amiodarone therapy was obtained.

\section{Classification of AF or Flutter}

Paroxysmal AF or flutter is characterized by recurrent episodes alternating with SR $!^{16}$ Episodes that lasted longer than $2 \mathrm{~min}$ and spontaneously reversed within 7 days without antiarrhythmic drug therapy or electrical cardioversion were classified as paroxysmal, ${ }^{17}$ and those requiring pharmacological or nonpharmacological cardioversion for termination were classified as persistent ${ }^{16}$ If the condition could not be terminated despite these treatments or if 
Table 1 Baseline Clinical Characteristics

\begin{tabular}{lc}
\hline \hline No. of patients & 108 \\
Age (years) & $55 \pm 13$ \\
M/F & $92 / 16$ \\
Atrial fibrillation or flutter & $36(33 \%)$ \\
$\quad$ Paroxysmal & $72(67 \%)$ \\
$\quad$ Nonparoxysmal & $103(95 \%)$ \\
Ventricular tachycardia or fibrillation & \\
Underlying heart disease & $37(34 \%)$ \\
$\quad$ Ischemic heart disease & $30(28 \%)$ \\
Idiopathic dilated cardiomyopathy & $8(7 \%)$ \\
Hypertrophic cardiomyopathy & $6(6 \%)$ \\
Arrhythmogenic right ventricular & \\
$\quad$ cardiomyopathy & $11(10 \%)$ \\
Valvular disease & $8(7 \%)$ \\
Congenital heart disease & $8(7 \%)$ \\
Others & IIs 14, IIm 75, III 10, IV 9 \\
NYHA functional class & $35 \pm 16$ \\
Left ventricular ejection fraction (\%) & \\
\hline
\end{tabular}

\pm values are mean $\pm S D$. Percentage are of patients.

patient did not wish to undergo cardioversion and remained in a state of AF, the case was classified as permanent $\mathrm{AF}$ or flutter; that is, labeling a patient as having permanent AF or flutter was an expression of the patient's intent rather than a description of the pathophysiology! 16

\section{Drug Dosing}

Patients were loaded with oral amiodarone at a dose of $400-800 \mathrm{mg} /$ day for 7-14 days. In some cases administration was begun at $200 \mathrm{mg} /$ day because of concomitant organic lung disease or reduced diffusing capacity of the lungs. After the initial loading phase, the maintenance dose of 50-200 mg/day was adjusted while the efficacy and side effects were monitored.

\section{Follow-up}

All patients were hospitalized for at least 2 weeks while undergoing the initial amiodarone loading and follow-up was continued at 1 month after discharge, and then at intervals of 1-3 months. Baseline 12-lead ECG, echocardiography, Holter monitoring, thyroid and liver function tests, pulmonary function tests, ophthalmologic examination, and chest X-ray were performed for most patients before amiodarone therapy (those requiring emergency care for acute myocardial infarction or severe heart failure did not always undergo Holter monitoring, pulmonary function testing or an ophthalmologic examination). Twelve-lead ECG was performed several times during the initial loading phase and also at each outpatient visit. Holter monitoring, thyroid and liver function testing, pulmonary function testing, and chest X-ray were used to assess the efficacy and adverse effects of amiodarone therapy at week 2, month 1 and 3 , and then every 3 months after initiation. Echocardiography and ophthalmologic examination were performed every 6 months.

We confirmed the recurrence of $\mathrm{AF}$ or flutter with or without symptoms by ECG at scheduled, unscheduled, or urgent visits, by Holter monitoring or by the recording of a device such as a pacemaker or implanted cardioverter defibrillator. We also investigated the possibility of recurrence when patients complained of typical clinical symptoms.

\section{Statistical Analysis}

Summary data are presented either as mean \pm SD or as

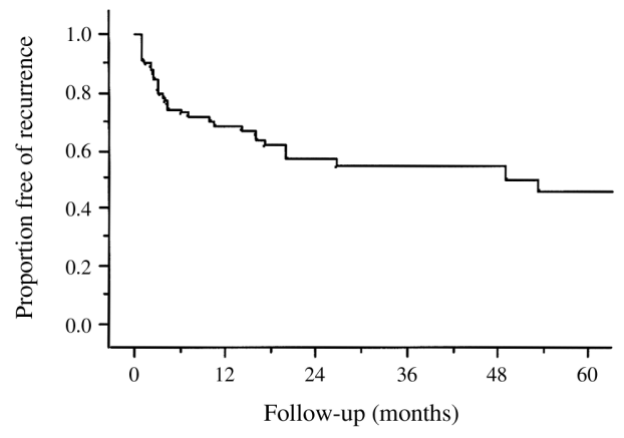

Fig 1. Kaplan-Meier estimates of the proportion of patients with no recurrence of atrial fibrillation or flutter in those with sinus rhythm at baseline or 1 month after initiation of amiodarone $(n=82)$.

numbers of patients. Baseline characteristics were compared between patients with and without recurrence of AF or flutter by either the Mann-Whitney test or Student's t-test. Mean heart rate values during Holter monitoring for each month after initiation of amiodarone therapy were compared with the baseline (before initiation) data by paired Student's t-test. The cumulative rate of recurrence of AF or flutter, and that for cases in permanent AF, were estimated by the product-limit method of Kaplan and Meier, and the differences among the various groups were assessed by the log-rank test. A p value less than 0.05 was considered statistically significant.

\section{Results}

\section{Patient Characteristics}

In this study, 72 patients $(67 \%)$ had either persistent or permanent $\mathrm{AF}$ or flutter and 36 had paroxysmal AF or flutter; 103 patients $(95 \%)$ had concomitant sustained or nonsustained ventricular tachycardia or ventricular fibrillation. Ischemic heart diseases accounted for $34 \%$ of the underlying heart diseases and nonischemic conditions accounted for $64 \%$. All 8 patients with hypertrophic cardiomyopathy had a history of congestive heat failure, and 7 patients were in the end-stage or dilated phase with concomitant systolic dysfunction! 18,19 The mean LVEF was $35 \pm 16 \%$ (Table 1 ).

Among the patients in this study, 37 (34\%) had received class I antiarrhythmic drugs prior to amiodarone therapy and in all but $2 \mathrm{AF}$ or flutter recurred in spite of treatment. Ventricular tachycardia also appeared as a drug-induced proarrhythmia in 2 other cases.

\section{Response to Amiodarone Therapy}

During a mean follow-up period of $36 \pm 28$ months (median: 25 ; range: $1-123$ months), 16 patients died: sudden cardiac death (4 patients), heart failure (6 patients), and noncardiac causes (6 patients).

The basal rhythm at the start of amiodarone therapy (baseline) was found to be AF in 39 patients. Sinus rhythm was successfully restored in 13 patients, including 7 patients by electrical cardioversion, within 1 month after initiation of amiodarone therapy. During the follow-up period, 34 $(41 \%)$ of the 82 patients who were in SR after 1 month of amiodarone administration had recurrence of AF or flutter. The time to first recurrence ranged from 1 to 28 months after initiation of amiodarone therapy, and 24 patients (71\%) experienced their first recurrence within 12 months 
Table 2 Clinical Characteristics of Patients With and Without Recurrence of Atrial Fibrillation or Flutter

\begin{tabular}{lccc}
\hline \hline & $\begin{array}{c}\text { With } \\
\text { recurrence }\end{array}$ & $\begin{array}{c}\text { Without } \\
\text { recurrence }\end{array}$ & p value \\
\hline No. of patients & 34 & 48 & \\
Age (years) & $55 \pm 15$ & $56 \pm 16$ & 0.69 \\
M/F & $27 / 7$ & $40 / 8$ & 0.65 \\
Ischemic heart disease & $8(24 \%)$ & $22(46 \%)$ & 0.03 \\
Left ventricular ejection fraction (\%) & $37 \pm 18$ & $35 \pm 16$ & 0.60 \\
Paroxysmal atrial fibrillation or flutter & $10(29 \%)$ & $26(54 \%)$ & 0.03 \\
Maintenance dose of amiodarone (mg/day) & $147 \pm 67$ & $132 \pm 48$ & 0.24 \\
Concomitant drugs & $22(65 \%)$ & $36(75 \%)$ & 0.32 \\
$\quad$ Diuretics & $21(62 \%)$ & $17(35 \%)$ & 0.02 \\
Digoxin & $21(62 \%)$ & $39(81 \%)$ & 0.15 \\
ACE inhibitors/AT1-blockers & $19(56 \%)$ & $22(46 \%)$ & 0.08 \\
B -adrenoceptor antagonists & $9(26 \%)$ & $20(42 \%)$ & 0.16 \\
Antiplatelet drugs & $22(65 \%)$ & $30(63 \%)$ & 0.63 \\
Anticoagulants & & & \\
\hline
\end{tabular}

\pm values are mean $\pm S D$. Percentage are of patients with or without recurrence. ACE, angiotensin-converting enzyme; AT1, angiotensin II subtype 1.

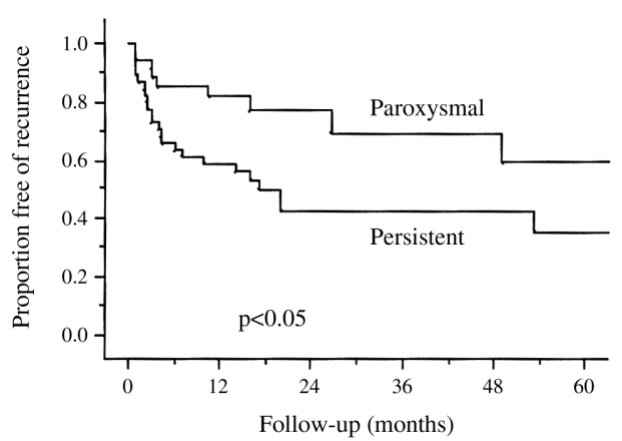

Fig 2. Kaplan-Meier estimates of the proportion of patients with no recurrence of atrial fibrillation or flutter in those with paroxysmal $(n=36)$ vs persistent $(n=46)$ atrial fibrillation or flutter.

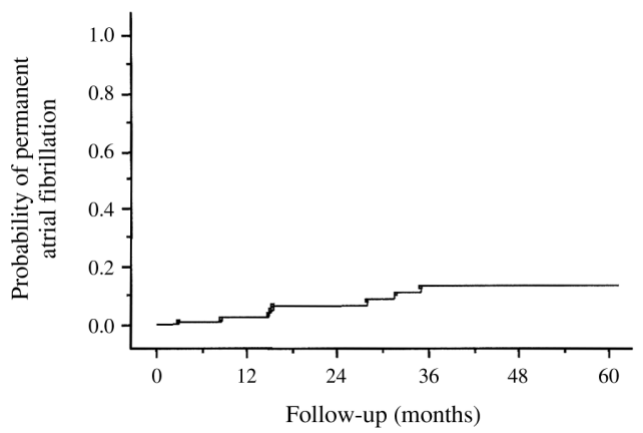

Fig 4. Kaplan-Meier estimates of the probability of permanent atrial fibrillation in patients with sinus rhythm at baseline or 1 month after beginning amiodarone $(n=82)$.

of initiation. The cumulative rates for maintenance of SR were $0.68,0.55$, and 0.47 at 1,3 , and 5 years, respectively (Fig 1). The maintenance dose of amiodarone was not significantly different between patients with and without recurrence. There were lower rates of ischemic heart disease and lower occurrence of paroxysms in patients with recurrence than in those without recurrence (Table 2). The cumulative rate of maintenance of SR was significantly higher in patients with paroxysmal AF or flutter than in those with the persistent form $(\mathrm{p}<0.05)$ (Fig 2), and there was a significantly greater rate of recurrence in patients with a left

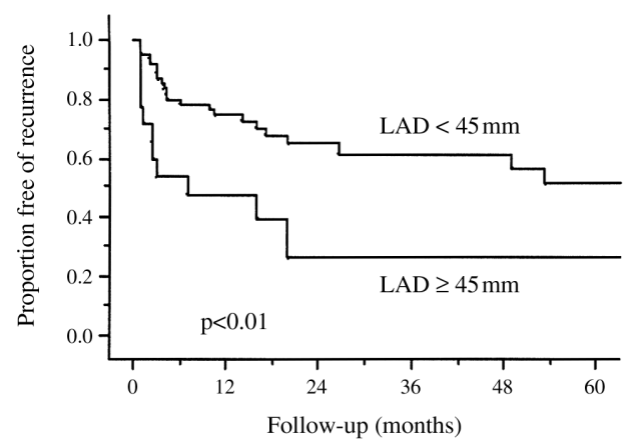

Fig 3. Kaplan-Meier estimates of the proportion of patients with no recurrence of atrial fibrillation or flutter in those with left atrial diameter $(\mathrm{LAD})<45 \mathrm{~mm}(\mathrm{n}=64)$ vs $\mathrm{LAD} \geq 45 \mathrm{~mm}(\mathrm{n}=18)$ at baseline.

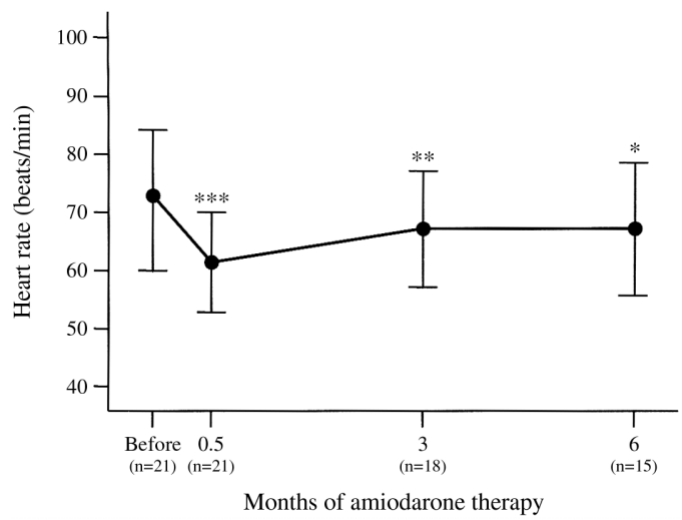

Fig 5. Changes in mean heart rate during Holter monitoring in patients with permanent atrial fibrillation before and during amiodarone therapy. Values are expressed as mean $\pm \mathrm{SD}$. $* * * \mathrm{p}<0.001$, $* * \mathrm{p}<0.01, * \mathrm{p}<0.05$ compared with before.

atrial diameter (LAD) $\geq 45 \mathrm{~mm}$ than in those with a LAD $<45 \mathrm{~mm}(\mathrm{p}<0.01)$ (Fig 3).

The cumulative rates for cases that remained in permanent $\mathrm{AF}$ during amiodarone therapy were $0.04,0.11$, and 0.14 at 1,3 , and 5 years, respectively (Fig 4 ). Permanent $\mathrm{AF}$ was found in 26 patients before and during amiodarone therapy and of those, 21 were examined to detect changes in mean heart rate by Holter monitoring within 6 months of 
initiating amiodarone therapy. They were also given the same doses of negative chronotropic drugs, such as digoxin and $\beta$-adrenoceptor antagonists, during this period and the mean heart rate significantly decreased after initiation of amiodarone therapy (Fig 5).

\section{Side Effects Requiring Discontinuation of Amiodarone}

Amiodarone therapy was discontinued in 17 patients (16\%): 10 patients had a decrease in the DLCo, an indicator of the diffusing capacity of the lung, 3 patients developed pulmonary fibrosis, 1 patient developed hyperthyroidism, 2 patients developed sinus bradyarrhythmia and 1 patient had sustained monomorphic ventricular tachycardia. None of the patients died as a result of the adverse effects associated with amiodarone.

\section{Discussion}

Amiodarone is effective in supraventricular arrhythmia as well as ventricular arrhythmia? The development of AF results in worsening heart failure, causing an uncontrolled heart rate with shortened filling time and provocation of tachycardiomyopathy; absence of the atrial kick and irregular ventricular rhythm will lead to a fall in cardiac output? Neurohormonal disturbance will also be induced by activation of the autonomic nervous system and renin-angiotensin system, and will in turn aggravate the heart failure and $\mathrm{AF}^{3}, 4 \mathrm{~A}$ recent report indicated that the plasma levels of atrial and brain natriuretic peptides could provide independent prognostic information of the recurrence of $\mathrm{AF}$ in patients with mild heart failure ${ }^{20}$ There is also a risk of thromboembolism in patients with uncontrolled heart failure who develop AF5 In Japanese patients with AF, underlying heart disease, particularly nonischemic cardiomyopathy, increases the risk of cerebral and peripheral arterial ischemic events. ${ }^{21}$ The maintenance of SR is important in $\mathrm{AF}$ in patients with impaired cardiac function resulting from structural heart disease.

Nonrandomized trials showed that the superiority of amiodarone in maintaining SR in cases of refractory $\mathrm{AF}$ or flutter in which SR could not be maintained with class I antiarrhythmic drugs, ${ }^{2-29}$ and a recent randomized trial showed that amiodarone is more effective than propafenone and sotalol for the prevention of AF 30 Thirty-seven of the present patients had been given class I antiarrhythmic drugs before the amiodarone therapy trial, but in most cases their AF could not be controlled. Moreover, class I antiarrhythmic drugs are associated with a higher incidence of adverse effects, such as proarrhythmia and conduction disturbances, and thus increase the risk of arrhythmic death or worsening heart failure $3,5,7$ In the present study, $70 \%$ of the patients were administered angiotensin-converting enzyme inhibitor or angiotensin II type 1 receptor blocker as the basic treatment of heart failure and these drugs may also be beneficial in the management of heart failure and $\mathrm{AF}$ through their reduction of neurohormonal activation!,3

Our result of $59 \%$ of patients successfully maintaining SR while on low-dose amiodarone during a mean followup of 18 months is comparable with the results of previous studies (53-79\%) ${ }^{22-30}$ However, unlike the previous studies in which recurrence increased linearly, our results are characterized by the first recurrence most often occurring within 1 year of initiating amiodarone therapy and the reasons for this difference may include: (1) a lower maintenance dose of amiodarone than previously reported (200-400 mg/day);29
(2) symptomatic heart failure and left ventricular dysfunction in all patients in the present study; and (3) many of the present patients had nonischemic heart disease. However, the final maintenance dose in patients without recurrence was $132 \pm 48 \mathrm{mg} /$ day (plasma concentrations were $0.54 \pm 0.30$ $\mu \mathrm{g} / \mathrm{ml}$ for amiodarone and $0.48 \pm 0.24 \mu \mathrm{g} / \mathrm{ml}$ for its active metabolite, desethylamiodarone), which was lower than previously reported, ${ }^{28-30}$ so we believe that the effect of amiodarone may not be simply a matter of the dose-effect relationship during long-term therapy.

In addition, it would appear that amiodarone is effective in maintaining SR as the basal rhythm because the rate of remaining in permanent $\mathrm{AF}$ was very low in the present study, even in cases of recurrence. To date, the efficacy of treatment for atrial tachyarrhythmia has been evaluated in clinical trials by comparing the time to the first recurrence; this method may be considerably less able to detect effective treatment than has been supposed 31 Previous reports have indicated that $40-50 \%$ of cases of heart failure result in sudden cardiac death,2,33 but in our study of patients receiving amiodarone, there were only 4 sudden cardiac deaths. Therefore, there is a need for a comprehensive evaluation of the efficacy of amiodarone for the prevention of not only atrial tachyarrhythmia but also sudden cardiac death in patients with heart failure.

Our results demonstrated that amiodarone was more effective in maintaining SR in cases of paroxysmal rather than in persistent $\mathrm{AF}$ or flutter, which differs from the results of Chun et al. ${ }^{28}$ The reason for the greater recurrence rate in patients with persistent AF is not clear, but may be related to the organically stabilized substrate. Atrial enlargement is considered both a cause and a consequence of atrial tachyarrhythmia and in the present study the incidence of recurrence was higher in cases with a large left atrial diameter $(\geq 45 \mathrm{~mm})$. Additionally, in terms of atrial stretch, atrial tachyarrhythmia is induced by activation of stretch-activated ion channels ${ }^{34}$ which induces abnormal automaticity and triggered activity, slowing of conduction, and increased dispersion of refractoriness $3,4,34$

Apart from suppression of $\mathrm{AF}$, amiodarone reduces the ventricular rate in permanent $\mathrm{AF}^{3,35}$ and also improves the hemodynamics of patients with rapid $\mathrm{AF}$ and heart failure 36 In the present study, the mean heart rate during Holter monitoring before and during amiodarone therapy was significantly decreased within 2 weeks of drug administration and the effect remained after 6 months in cases of permanent $\mathrm{AF}$, and this may play a part in the beneficial effect of long-term amiodarone therapy.

\section{Adverse Effects}

The incidence of intolerable noncardiac effects resulting in withdrawal of the drug in low-dose amiodarone therapy for $\mathrm{AF}$ has been reported as $1-21 \% 22-30 \mathrm{We}$ did not record any fatal adverse events, but amiodarone was discontinued because of noncardiac effects in 14 cases (13\%), a rate similar to that in the other reports.

\section{Study Limitations}

This study was retrospective without a control group, and was not always designed to study the protocol for the prevention of atrial tachyarrhythmia. In addition, cardioversion was not performed in all cases showing AF at baseline, and the study lacked objectivity in the definition of permanent AF. Therefore, we cannot make a conclusion about the absolute efficacy of low-dose amiodarone for suppression 
of AF.

\section{Conclusion}

We retrospectively examined the efficacy and safety of amiodarone in maintaining SR in Japanese patients with AF or flutter and heart failure. It would appear to be effective, with few cardiac side effects, and may also achieve ventricular rate control in cases of permanent AF. Therefore, clinicians can expect that amiodarone therapy for Japanese patients with atrial tachyarrhythmia and concomitant heart failure will be efficacious.

\section{References}

1. Stevenson WG, Stevenson LW, Middekauff HR, Fonarow GC, Hamilton MA, Woo MA, et al. Improving survival for patients with atrial fibrillation and advanced heart failure. J Am Coll Cardiol 1996; 28: $1458-1463$.

2. Benjamin EJ, Wolf PA, D'Agostino RB, Silbershatz H, Kannel WB, Levy D. Impact of atrial fibrillation on the risk of death: The Framingham Heart Study. Circulation 1998; 98: 946-952.

3. Crijns HJGM, Van Den Berg MP, Van Gelder IC, Van Veldhuisen DJ. Management of atrial fibrillation in the setting of heart failure. Eur Heart J 1997; 18: C45-C49.

4. Van Den Berg MP, Tuinenburg AE, Crijns HJGM, Van Gelder IC, Gosselink ATM, et al. Heart failure and atrial fibrillation: Current concepts and controversies. Heart 1997; 77: 309-313.

5. The Stroke Prevention in Atrial Fibrillation Investigators. Predictors of thromboembolism in atrial fibrillation. Ann Intern Med 1992; 116: $1-5$.

6. Middlekauff HR, Stevenson WG, Stevenson LW. Prognostic significance of atrial fibrillation in advanced heart failure: A study of 390 patients. Circulation 1991; 84: 40-80.

7. Flaker GC, Blackshear JL, McBride R, Kronmal RA, Halperin JL, Hart RG. Antiarrhythmic drug therapy and cardiac mortality in atrial fibrillation: The Stroke Prevention in Atrial Fibrillation Investigators. J Am Coll Cardiol 1992; 20: 527-532.

8. Coplen SE, Antman EM, Berlin JA, Hewitt P, Chalmers TC. Efficacy and safety of quinidine therapy for maintenance of sinus rhythm after cardioversion: A meta-analysis of randomized control trials. Circulation 1990; 82: 1106-1116.

9. Singh BN. Current antiarrhythmic drugs: An overview of mechanisms of action and potential clinical utility. J Cardiovasc Electrophysiol 1999; 10: 283-301.

10. Singh SN, Fletcher RD, Fisher SG, Singh BN, Lewis HD, Deedwania $\mathrm{PC}$, et al for the Survival Trial of Antiarrhythmic Therapy in Congestive Heart Failure. Amiodarone in patients with congestive heart failure and asymptomatic ventricular arrhythmia. $N$ Engl J Med 1995; 333: 77-82.

11. Deedwania PC, Singh BN, Ellenbogen K, Fisher S, Fletcher R, Singh $\mathrm{SN}$, for the Department of Veterans Affairs CHF-STAT Investigators. Spontaneous conversion and maintenance of sinus rhythm by amiodarone in patients with heart failure and atrial fibrillation: Observation from the Veterans Affairs Congestive Heart Failure Survival Trial of Antiarrhythmic Therapy (CHF-STAT). Circulation 1998; 98: 25742579.

12. Doval HC, Nul DR, Grancelli HO, Perrone SV, Bortman GR, Curiel $\mathrm{R}$, for Grupo de Estudio de la Sobrevida en la Insuficiencia Cardiaca en Argentina (GESICA). Randomised trial of low-dose amiodarone in severe congestive heart failure. Lancet 1994; 344: 493-498.

13. Pratt C, Waldo AL, Camm AJ. Can antiarrhythmic drugs survive survival trials? Am J Cardiol 1998; 81: 24D-34D.

14. Prystowsky EN. Management of atrial fibrillation: Therapeutic options and clinical decisions. Am J Cardiol 2000; 85: 3D-11D.

15. Torp-Pedersen C, Mller, Bloch-Thomsen PE, Kber L, Sande E, Egstrup K, et al for the Danish Investigations of Arrhythmia and
Mortality on Dofetilide Study Group. Dofetilide in patients with congestive heart failure and left ventricular dysfunction. $N$ Engl $J$ Med 1999; 34: 857-865.

16. Gallanger MM, Camm AJ. Classification of atrial fibrillation. Pacing Clin Electrophysiol 1997; 20: $1603-1605$.

17. Lévy S, Novella P, Ricard P, Paganelli P. Paroxysmal atrial fibrillation. A need for classification. J Cardiovasc Electrophysiol 1995; 6: $69-74$.

18. Fujiwara H, Onodera T, Tanaka M, Shirane H, Kato H, Yoshikawa J, et al. Progression from hypertrophic obstructive cardiomyopathy to typical dilated cardiomyopathy-like feature in the end stage. Jpn Circ $J$ 1984; 48: 1210-1214.

19. Maron BJ, Spirito P. Implications of left ventricular remodeling in hypertrophic cardiomyopathy. Am J Cardiol 1998; 81: 1339-1344.

20. Mabuchi N, Tsutamoto T, Maeda K, Kinoshita M. Plasma cardiac natriuretic peptides as biochemical markers of recurrence of atrial fibrillation in patients with mild congestive heart failure. Jpn Circ J 2000; 64: 765-771.

21. Tomita F, Kohya T, Sakurai M, Kaji T, Yokoshiki H, Sato M, et al for the Hokkaido Atrial Fibrillation Study Group. Prevalence and clinical characteristics of patients with atrial fibrillation: Analysis of 20,000 cases in Japan. Jpn Circ J 2000; 64: 653-658.

22. Brodsky MA, Allen BJ, Walker III CJ, Casey TP, Luckett CR, Henry WL. Amiodarone for maintenance of sinus rhythm after conversion of atrial fibrillation in the setting of a dilated left atrium. Am J Cardiol 1987; 60: $572-575$.

23. Gold RL, Haffajee CI, Charos G, Sloan K, Baker S, Alpert JS. Amiodarone for refractory atrial fibrillation. Am J Cardiol 1986; 57: $124-127$.

24. Graboys TB, Podrid PJ, Lown B. Efficacy of amiodarone for refractory supraventricular tachyarrhythmias. Am Heart J 1983; 106: 870876.

25. Horowitz LN, Spielman SR, Greenspan AM, Mintz GS, Morganroth $\mathrm{J}$, Brown R, et al. Use of amiodarone in the treatment of persistent and paroxysmal atrial fibrillation resistant to quinidine therapy. $\mathrm{J} \mathrm{Am}$ Coll Cardiol 1985; 6: 1402-1407.

26. Blevins RD, Kerin NZ, Benaderet D, Frumin H, Faitel K, Jarandilla $\mathrm{R}$, et al. Amiodarone in the management of refractory atrial fibrillation. Arch Intern Med 1987; 147: 1401-1404.

27. Grosselink ATM, Crijns HJGM, Van Gelder IC, Hillige H, Wiesfeld ACP, Lie KI. Low-dose amiodarone for maintenance of sinus rhythm after cardioversion of atrial fibrillation or flutter. JAMA 1992; 267: 3289-3293.

28. Chun SH, Sagar PT, Stevenson WG, Nademanee K, Middlekauff HR, Singh BN. Long-term efficacy of amiodarone for the maintenance of normal sinus rhythm in patients with refractory atrial fibrillation or flutter. Am J Cardiol 1995; 76: 47-50.

29. Middlekauff HR, Wiener I, Stevenson WG. Low-dose amiodarone for atrial fibrillation. Am J Cardiol 1993; 72: 75F-81F.

30. Roy D, Talajic M, Dorian P, Connolly S, Eisenberg MJ, Green M, for the Canadian Trial of Atrial Fibrillation investigators. Amiodarone to prevent recurrence of atrial fibrillation. $N$ Engl J Med 2000; 342: 913-920.

31. Kaemmerer WF, Rose MS, Mehra R. Distribution of patients' paroxysmal atrial tachyarrhthmia episodes: Implication for detection of treatment efficacy. J Cardiovasc Electrophysiol 2001; 12: 121-130.

32. Packer M. Sudden unexpected death in patients with congestive heart failure: A second frontier. Circulation 1985; 72: 681-685.

33. Kannel WB, Plehn JF, Cupples LA. Cardiac failure and sudden death in the Framingham study. Am Heart J 1989; 115: 869-875.

34. Bode F, Sachs F, Franz MR. Tarantula peptide inhibits atrial fibrillation: A peptide from spider venom can prevent the heartbeat from losing its rhythm. Nature $2001 ; \mathbf{4 0 9}: 35-36$.

35. Tse HF, Lam YM, Lau CP, Cheung BM, Kumana CR. Comparison of digoxin versus low-dose amiodarone for ventricular rate control in patients with chronic atrial fibrillation. Clin Exp Pharmacol Physiol 2001; 28: 446-450.

36. Clemo HF, Wood MA, Gilligan DM, Ellenbogen KA. Intravenous amiodarone for acute heart rate control in the critically ill patient with atrial tachyarrhythmias. Am J Cardiol 1998; 81: 594-598. 